\title{
Revisiting The Averaged Problem in The Case of Mean-Motion Resonances of The Restricted Three- Body Problem. Global Rigorous Treatment and Application To The Co-Orbital Motion.
}

\author{
Alexandre Pousse ( $\square$ alexandre@mi.imati.cnr.it ) \\ IMATI CNR Milano: Istituto di Matematica Applicata e Tecnologie Informatiche Enrico Magenes \\ Consiglio Nazionale delle Ricerche Sezione di Milano \\ Elisa Maria Alessi \\ IMATI CNR Milano: Istituto di Matematica Applicata e Tecnologie Informatiche Enrico Magenes \\ Consiglio Nazionale delle Ricerche Sezione di Milano
}

\section{Research Article}

Keywords: Restricted three-body problem, Perturbative treatment, Averaged Hamiltonian, Mean-motion resonance, Co-orbital motion

Posted Date: July 1st, 2021

DOl: https://doi.org/10.21203/rs.3.rs-614015/v1

License: (c) (i) This work is licensed under a Creative Commons Attribution 4.0 International License.

Read Full License

Version of Record: A version of this preprint was published at Nonlinear Dynamics on February 4th, 2022. See the published version at https://doi.org/10.1007/s11071-022-07229-5. 


\section{Abstract}

A classical approach to the restricted three-body problem is to analyze the dynamics of the massless body in the synodic reference frame. A different approach is represented by the perturbative treatment: in particular the averaged problem of a mean-motion resonance allows to investigate the long-term behavior of the solutions through a suitable approximation that focuses on a particular region of the phase space. In this paper, we intend to bridge a gap between the two approaches in the specific case of mean-motion resonant dynamics, establish the limit of validity of the averaged problem, and take advantage of its results in order to compute trajectories in the synodic reference frame. After the description of each approach, we develop a rigorous treatment of the averaging process, estimate the size of the transformation and prove that the averaged problem is a suitable approximation of the restricted threebody problem as long as the solutions are located outside the Hill's sphere of the secondary. In such a case, a rigorous theorem of stability over finite but large timescales can be proven. We establish that a solution of the averaged problem provides an accurate approximation of the trajectories on the synodic reference frame within a finite time that depend on the minimal distance to the Hill's sphere of the secondary. The last part of this work is devoted to the co-orbital motion (i.e., the dynamics in 1:1 meanmotion resonance) in the circular-planar case. In this case, an interpretation of the solutions of the averaged problem in the synodic reference frame is detailed and a method that allows to compute coorbital trajectories is displayed.

\section{Full Text}

This preprint is available for download as a PDF. 